\title{
Whole-genome sequencing of glioblastoma reveals enrichment of non-coding constraint mutations in known and novel genes
}

Sharadha Sakthikumar ${ }^{1,2+}$, Ananya Roy ${ }^{3+}$, Lulu Haseeb ${ }^{3+}$, Mats E. Pettersson ${ }^{1}$, Elisabeth Sundström ${ }^{1}$, Voichita D. Marinescu', Kerstin Lindblad-Toh ${ }^{1,2}$ and Karin Forsberg-Nilsson ${ }^{3^{*}}$ (I)

\author{
*Correspondence: karin.nilsson@ \\ igp.uu.se \\ 'Sharadha Sakthikumar, Ananya Roy \\ and Lulu Haseeb contributed \\ equally to this work. \\ ${ }^{3}$ Department of Immunology, \\ Genetics and Pathology, Science for \\ Life Laboratory, Uppsala University, \\ SE-751 85 Uppsala, Sweden \\ Full list of author information is \\ available at the end of the article
}

\begin{abstract}
Background: Glioblastoma (GBM) has one of the worst 5-year survival rates of all cancers. While genomic studies of the disease have been performed, alterations in the non-coding regulatory regions of GBM have largely remained unexplored. We apply whole-genome sequencing (WGS) to identify non-coding mutations, with regulatory potential in GBM, under the hypothesis that regions of evolutionary constraint are likely to be functional, and somatic mutations are likely more damaging than in unconstrained regions.

Results: We validate our GBM cohort, finding similar copy number aberrations and mutated genes based on coding mutations as previous studies. Performing analysis on non-coding constraint mutations and their position relative to nearby genes, we find a significant enrichment of non-coding constraint mutations in the neighborhood of 78 genes that have previously been implicated in GBM. Among them, SEMA3C and DYNC1/1 show the highest frequencies of alterations, with multiple mutations overlapping transcription factor binding sites. We find that a non-coding constraint mutation in the SEMA3C promoter reduces the DNA binding capacity of the region. We also identify 1776 other genes enriched for non-coding constraint mutations with likely regulatory potential, providing additional candidate GBM genes. The mutations in the top four genes, DLX5, DLX6, FOXA1, and ISL1, are distributed over promoters, UTRs, and multiple transcription factor binding sites
\end{abstract}

Conclusions: These results suggest that non-coding constraint mutations could play an essential role in GBM, underscoring the need to connect non-coding genomic variation to biological function and disease pathology.

Keywords: Glioblastoma, Cancer, Non-coding constraint, Gene regulation 


\section{Background}

Glioblastoma (GBM) is an extremely aggressive brain tumor, characterized by high interand intra-patient heterogeneity $[1,2]$. Despite maximal safe resection, followed by radiotherapy and chemotherapy with temozolomide (TMZ), the average survival is only 15 months [3]. Two forms of GBM are defined based on genetic mutations observed in the isocitrate dehydrogenase (IDH1 and IDH2) genes [4]. Primary GBM comprises $90 \%$ of the cases and is isocitrate dehydrogenase $I D H$-wild-type, while secondary GBM develops from lower grade glioma and carry mutations in IDH. Although these two types of GBM are histologically indistinguishable, they differ in genetic and clinical features [5]. Common genetic alterations in GBM include loss of the chromosome arm 10q, alterations in TP53 and RB, amplifications of EGFR and PDGFR, and aberrations in RTK/Ras/PI3K signaling pathways, all of which are major known drivers of GBM pathology. Other frequent mutations include alterations in NF1, PTEN, and MDM2 [6, 7]. GBMs are continually evolving and, within a single patient, could display multiple subtypes, gene profiles, transcriptome patterns, and methylation phenotypes, all features that could favor sub-clonal selection [8]. Based on extensive molecular classification of GBM by "The Cancer Genome Atlas" (TCGA), distinct molecular subtypes for IDH-wild-type GBMs were identified [7, 8].

Whole-genome sequencing (WGS) of GBM tumors has highlighted the importance of TERT promoter mutations in the development of the disease [9] and has been instrumental in improved understanding of clonal and sub-clonal evolution for GBM recurrences [10]. Because the absolute majority of mutations in cancer reside in the non-coding part of the genome [11], WGS also paves the way for further identification of mutations in regulatory elements such as promoters and enhancers. Evolutionary constraint across species is one of the chief indications of functional potential and has been studied across mammals [12] and vertebrates [13]. In addition, multiple other resources exist to assign function, including non-coding RNAs [14], enhancer predictions [15], ENCODE data of transcription factor binding sites, histone methylation, and DNA methylation [16]. Many of these changes accumulate in the genome all along the oncogenic process and are likely genetic or epigenetic adaptations that are prone to be conserved for a functional outcome [11].

We have performed WGS of 38 matched tumor tissue and corresponding blood samples from GBM patients, to identify novel somatic variants in regulatory regions in and around GBM genes. The catalog of exome mutations across our samples largely mirrors what has previously been described, thus serving to validate the cohort. In addition, we report here a range of non-coding constraint mutations (NCCM) that may have functional impact on the disease. Our data provides evidence of enrichment of NCCMs for GBMassociated genes, including SEMA3C and DYNC111, as well as in more than 1776 other genes, many of which have not previously been linked to GBM. We therefore surmise that a better understanding of the non-coding genome of GBM tumors will help in the elucidation of functional genetic and epigenetic alterations and consequently may unlock therapeutic opportunities for personalized treatment strategies.

\section{Results}

Patient cohort and whole-genome sequencing of matched tumor and normal pairs The cohort designated SweGBM-1 comprised 39 IDH1 ${ }^{\text {wt }}$ GBM patients: 35 patients who underwent their first surgery (treatment naïve) and four patients whose tumor had 
recurred. Molecular subtypes for 37 of the 39 tumors included 15 classical, 15 mesenchymal, and 7 proneural types. For two of the samples, the type could not be characterized unambiguously (Fig. 1a and Table 1). To discover somatic alterations in the SweGBM-1 cohort, matched tumor/normal pairs $(n=39)$ were sequenced using Illumina whole-genome sequencing. Alignment of the sequencing reads to the

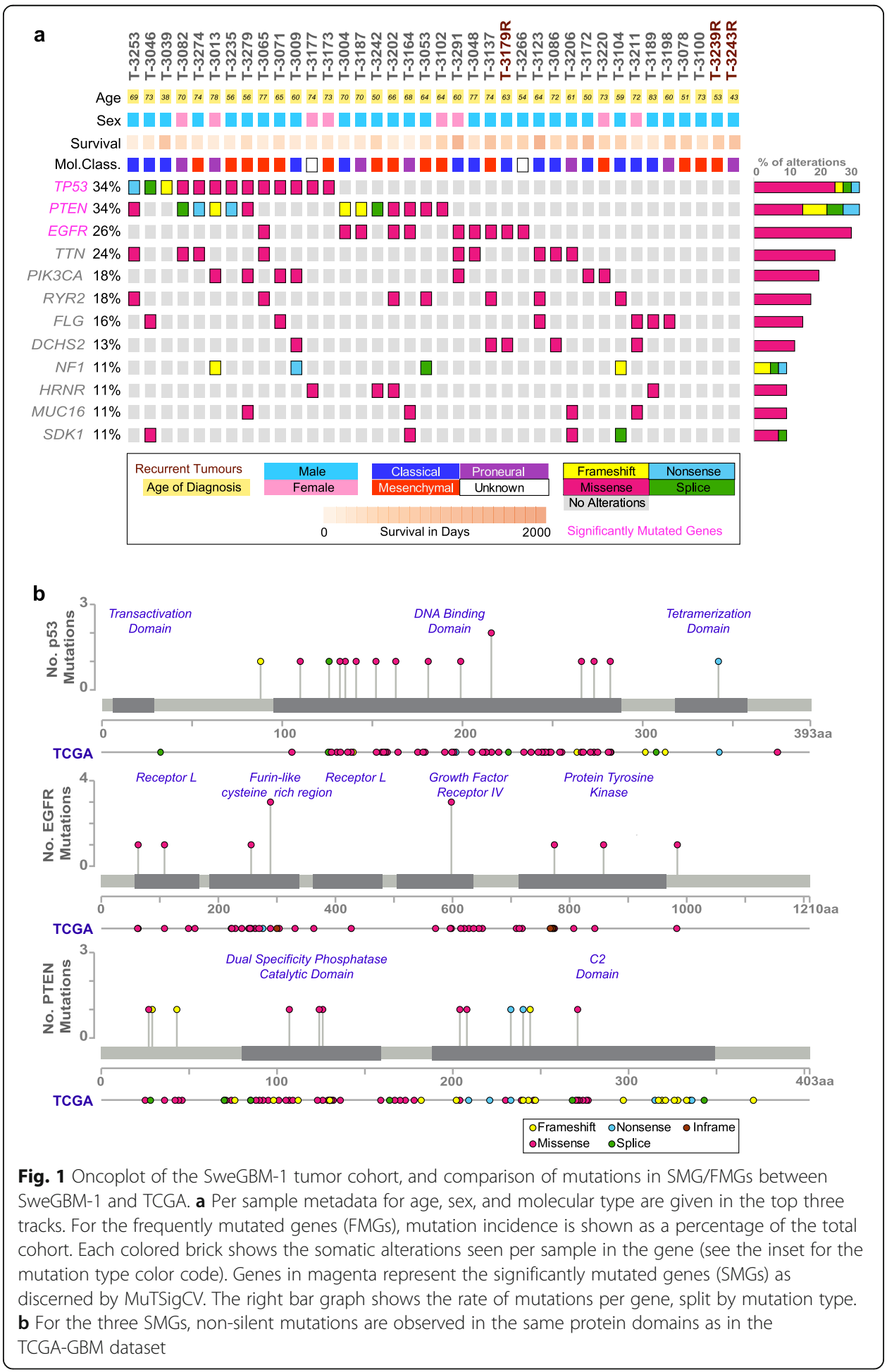


Table 1 Summary of SweGBM-1 $(n=39)$ cohort with somatic point and indel mutations statistics

\begin{tabular}{|c|c|c|c|c|c|c|c|c|c|}
\hline HGCC-ID & $\begin{array}{l}\text { Glioma } \\
\text { Grade* }\end{array}$ & Age & Sex & $\begin{array}{l}\text { Survival days } \\
\text { (diagnosis to } \\
\text { death) }\end{array}$ & $\begin{array}{l}\text { Subtype } \\
\text { classification } \\
\text { of the cell line }\end{array}$ & $\begin{array}{l}\text { Tumor purity } \\
\text { (aberrant cell } \\
\text { fraction) }\end{array}$ & $\begin{array}{l}\text { Depth of } \\
\text { coverage }\end{array}$ & $\begin{array}{l}\text { MuTect2- } \\
\text { Strelka } \\
\text { concordant } \\
\text { SPM }\end{array}$ & $\begin{array}{l}\text { MuTect2- } \\
\text { Strelka } \\
\text { concordant } \\
\text { SIM }\end{array}$ \\
\hline 3004 & IV & 70 & $M$ & 134 & $\mathrm{CL}$ & 89 & 72 & 7788 & 330 \\
\hline 3009 & IV & 60 & M & 174 & $\mathrm{CL}$ & 72 & 75 & 9037 & 359 \\
\hline 3013 & IV & 78 & $\mathrm{~F}$ & 122 & PN & 83 & 78 & 8477 & 308 \\
\hline 3039 & IV & 38 & M & 994 & $\mathrm{CL}$ & 98 & 77 & 7167 & 266 \\
\hline 3046 & IV & 73 & M & 186 & $\mathrm{CL}$ & 71 & 74 & 8587 & 336 \\
\hline 3048 & IV & 77 & M & 279 & $\mathrm{CL}$ & 99 & 72 & 9266 & 431 \\
\hline 3053 & IV & 64 & M & 277 & MS & 64 & 72 & 4201 & 157 \\
\hline 3065 & IV & 77 & M & 127 & MS & 84 & 86 & 14,666 & 450 \\
\hline 3071 & IV & 65 & M & 309 & MS & 82 & 70 & 9461 & 386 \\
\hline 3078 & IV & 51 & M & 716 & MS & 68 & 79 & 3729 & 170 \\
\hline 3082 & IV & 70 & F & 314 & PN & 95 & 89 & 10,122 & 385 \\
\hline 3086 & IV & 72 & M & 444 & $\mathrm{CL}$ & 86 & 72 & 5850 & 373 \\
\hline 3100 & IV & 73 & M & 284 & $\mathrm{CL}$ & 95 & 68 & 5028 & 74 \\
\hline 3102 & IV & 64 & F & 543 & MS & 96 & 75 & 7456 & 285 \\
\hline 3104 & IV & 59 & M & 585 & $\mathrm{CL}$ & 54 & 73 & 9661 & 347 \\
\hline 3123 & IV & 64 & M & 1613 & $\mathrm{CL}$ & 94 & 79 & 12,224 & 364 \\
\hline 3137 & IV & 74 & M & 1088 & MS & 46 & 78 & 5819 & 227 \\
\hline $3151^{\dagger}$ & IV & 61 & M & 817 & MS & 73 & 67 & 230,968 & 1562 \\
\hline 3164 & IV & 68 & M & 54 & PN & 91 & 81 & 8661 & 439 \\
\hline 3172 & IV & 50 & M & 1352 & $\mathrm{CL}$ & 78 & 78 & 4312 & 164 \\
\hline 3173 & IV & 73 & $\mathrm{~F}$ & 229 & MS & 51 & 64 & 5345 & 211 \\
\hline 3177 & IV & 74 & F & 179 & $\mathrm{n} / \mathrm{a}$ & 99 & 78 & 10,585 & 522 \\
\hline $3179^{\dagger}$ & IV & 63 & M & 474 & $\mathrm{CL}$ & 64 & 69 & 4696 & 325 \\
\hline 3187 & IV & 70 & M & 193 & PN & 62 & 73 & 4481 & 194 \\
\hline 3189 & IV & 83 & M & 218 & $\mathrm{CL}$ & 98 & 75 & 7710 & 444 \\
\hline 3198 & IV & 60 & M & 729 & PN & 75 & 78 & 7968 & 308 \\
\hline 3202 & IV & 66 & M & 137 & MS & 84 & 67 & 5361 & 324 \\
\hline 3206 & IV & 61 & M & 767 & PN & 71 & 83 & 4025 & 246 \\
\hline 3211 & IV & 72 & F & 206 & $\mathrm{CL}$ & 61 & 75 & 5151 & 265 \\
\hline 3220 & IV & 73 & F & 492 & MS & 70 & 79 & 4447 & 268 \\
\hline $3233^{+}$ & IV & 53 & M & 809 & MS & 100 & 68 & 1590 & 189 \\
\hline 3235 & IV & 56 & M & 141 & MS & 94 & 71 & 4570 & 203 \\
\hline 3242 & IV & 50 & $M$ & 449 & MS & 91 & 85 & 4336 & 246 \\
\hline $3243^{+}$ & IV & 43 & $M$ & 1070 & PN & 41 & 81 & 828 & 296 \\
\hline 3253 & IV & 69 & $M$ & 92 & $\mathrm{CL}$ & 79 & 78 & 8079 & 358 \\
\hline 3266 & IV & 54 & $M$ & 571 & $\mathrm{n} / \mathrm{a}$ & 85 & 76 & 4767 & 259 \\
\hline 3274 & IV & 74 & $M$ & 206 & MS & 68 & 69 & 6447 & 227 \\
\hline 3279 & IV & 56 & $M$ & 333 & MS & 41 & 78 & 6320 & 64 \\
\hline 3291 & IV & 60 & $F$ & 1527 & $\mathrm{CL}$ & 89 & 79 & 7782 & 327 \\
\hline
\end{tabular}

*All of the samples in the SweGBM-1 cohort are IDH ${ }^{\text {wt }}$

${ }^{\dagger}$ Recurrent tumors 
reference assembly hg19 [17] yielded depths of coverage of median $75 \times$ (range 64-89) for the tumors and $38 \times$ (range 30-66) for the matched normal.

\section{Somatic copy number alterations agree with the TCGA cohort}

Using the algorithm ascatNgs [18] to identify somatic copy number alterations (SCNA) present in the genome of the tumor sample relative to the matched normal, we analyzed the highly rearranged GBM landscape and found, on average, that $600 \mathrm{Mbp}$ was amplified and $770 \mathrm{Mbp}$ deleted per sample. The most widespread amplifications were seen in chromosome 7, covering the EGFR locus, in 99\% of the samples. In chromosome 10, comprising the PTEN locus, deletions were observed in $>90 \%$ of all samples. Overall, the SCNA patterns are highly similar to what was seen in the TCGA cohort (Additional file 1: Figure S1 a, b [19, 20];).

\section{Variant calling and functional annotation}

With the refined alignment BAM files, somatic point mutations (SPM) and somatic indel mutations (SIM) were called using MuTect2 [21] and Strelka [22]. To ensure that we had a high-quality dataset with few false-positive calls, only variants concordant between the two tools were used for the downstream analyses. A total of 256,000 SPMs and 11,127 SIMs were obtained across 38 samples (sample 3151 was hyper-mutated with 232,530 variants and hence was excluded from the above summary). Oncotator [23] was subsequently used for translational annotation of the somatic variants into coding and non-coding variant classes. Parsing the annotations for all the samples showed that $>98 \%$ of the variants were distributed across the non-coding part of the genome (Additional file 1: Figure S2a). Variant allele fraction (VAF, the fraction of reads overlapping a genomic coordinate that supports the alternate allele), however, showed no skew in the distribution for the coding and non-coding variant class categories (Additional file 1: Figure S2b).

\section{Protein-modifying alterations recapitulate annotations in TCGA GBM genes}

Among the coding variants, MuTSigCV [24] was used to delineate significantly mutated genes (SMGs, genes that harbor more protein-modifying variants than expected by chance and are more likely to play an active role in tumorigenesis). The algorithm identified three genes, TP53, EGFR, and PTEN as SMGs. Applying a frequency-based approach, we identify nine more frequently mutated genes (FMGs), with non-silent variants that were found in $\geq 4$ (10\%) of all samples (Fig. 1a). All 12 of the FMGs/SMGs overlap with the top 20 genes seen to be mutated in the TCGA GBM dataset (Additional file 2: Table S1 [25];). In addition, 21\% of mutations in the 12 proteins, encoded by these genes, are concordant with positions previously reported to have mutations in GBM (Fig. 1b, Additional file 3: Table S2). Also, 39\% of the altered positions seen in these genes, including TP53, EGFR, and PTEN, have not been previously seen in the TCGA GBM dataset, but have been observed in other cancers [26, 27] and/or are included in the Memorial Sloan Kettering (MSK) cancer hotspot resource [28] (Additional file 3: Table S2).

\section{TERT promoter mutations}

Frequent alterations in the promoter of the telomerase reverse transcriptase (TERT) gene have been described across several cancers $[29,30]$ including glioma $[9,31]$. In 
the SweGBM-1 dataset, relative to the TERT gene start codon, $>75 \%$ of samples are observed to have mutually exclusive mutations at - 124 bp, hg19 chr5:1,295,228 C > T (24/38 samples) and at - 146 bp (hg 19 chr5, 1,295,250 C > T (4/38 samples) (Additional file 1: Figure S3). These two mutational hotspots are also seen across a multitude of cancers [32]. In our study cohort, $>75 \%$ tumors have these mutations, which is close to the $80 \%$ observed by Heidenreich et al. [31], but less than what was seen by Korber et al. [9], wherein they note that all of their primary GBM tumors have one or the other hotspot changes. Heidenreich and colleagues [31] have also shown that for primary GBMs, there is an inverse correlation between mutations in the therapeutic/diagnostic markers of isocitrate dehydrogenase 1 (IDH1) and alterations in the above promoter coordinates of TERT. Since all of our tumor samples are IDH $1^{w t}$, observation of the above TERT promoter mutations in $76 \%$ of the patients is consistent with previous results.

\section{Enrichment of non-coding constraint mutations in the neighborhood of key GBM genes}

The majority of somatic mutations across tumor samples are found in the non-coding regions of the genome, consistent with the fact that $>98 \%$ of the genome is noncoding. Most of these alterations are likely neutral passenger events and are not expected to impact the fitness of cancer cells. Nonetheless, a fraction of these non-coding changes are associated with regulatory elements of specific genes, such as promoters, UTRs, splice signals, lncRNAs and transcription factor binding sites, enhancers, and DNA methylation regions that can be expected to have roles in tumorigenesis [33]. We, therefore, decided to investigate alterations in the regulatory sequences in the vicinity of a set of genes that have roles in GBM by surveying for mutations occurring in evolutionarily constraint sequences. Accordingly, we first selected 78 key genes, known to have frequent protein-coding changes in GBM by combining the SweGBM-1 SMG/FMG and TCGA-GBM SMG gene sets (Additional file 4: Table S3).

Roughly $80 \%$ of genetic variants that influence gene regulation occur in cis-acting eQTLs that reside within $1 \mathrm{Mbp}$ from their target genes [34-36]. Here, to be conservative, we applied a more stringent threshold and chose to examine somatic changes in intergenic regions $( \pm 100 \mathrm{kbp})$ of the above key genes together with mutations in UTRs, introns, and non-coding RNAs. We limited this search with the 33-vertebrate constraint [13], to target variants with potential functional impact. The data thus obtained were denominated as non-coding constraint mutations (NCCMs). We used the tool GERP++ [13] for the detection of NCCM in our data set (Fig. 2a). Variants with a GERP RS value $\geq 2$ were deemed to lie in constrained regions [13], and in our cohort, $\sim 15 \%$ of variants (both coding and non-coding) satisfied this criterion. Figure $2 \mathrm{~b}$ shows the distribution of variants in conserved sites, by category, for coding and non-coding regions.

We then evaluated the rate of NCCMs, normalized to the length of the queried genomic region for each of the key genes versus all other protein-coding genes (OPCG). An enrichment of NCCMs was observed in the neighborhoods of the key genes as compared to the frequency of NCCMs seen in the OPCGs (Fig. 3a, $t$ test, $P$ value < 0.0001). We also confirmed that the rates of non-coding constraint sites (positions that have the potential to become mutated) were uniform across the categories of key genes 


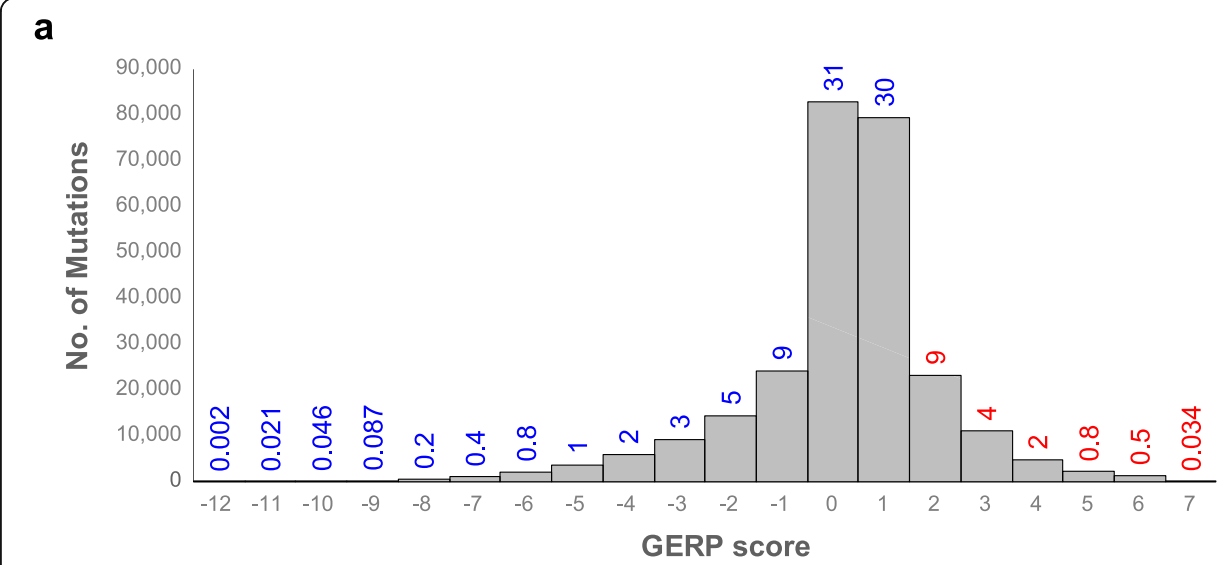

b
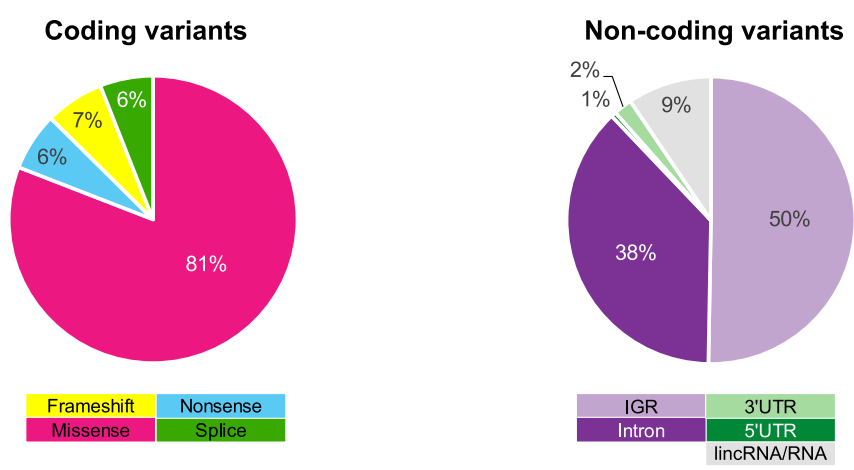

Fig. 2 GERP score distribution for the SweGBM-1 cohort. a Scores range from -12 to +7 ; approximately $15 \%$ of all variants, with GERP RS $\geq 2$ are deemed to be "constrained" (marked in red). b Distribution of variants in constrained sites by category for coding and non-coding mutations (see the inset for the mutation type color code)

and OPCGs (Additional file 1: Figure S4, $t$ test, $P$ value $=0.09$ ). This ensures that the enrichment we discerned is not a function of having a higher frequency of constraint sites in the flanking regions of our key genes. Of the 78 key genes, a total of 26 genes had $>1.0$ NCCM per $100 \mathrm{kbp}$ (Fig. 3b). Eight genes had constrained mutational frequencies that were $>2.0 / 100 \mathrm{kbp}$ (Fig. 3c), and the four genes with the most NCMMs per 100-kbp sequence were SEMA3C, DYNC1I1, LRFN5, and CNTNAP2.

Comprehensive annotation of the NCCMs, encompassing either reported or validated regulatory elements, or both, was carried out with data from the ENCODE Analysis Hub [37] and the UCSC Genome Browser [16] (Additional file 5: Table S4). A data-driven estimate of regulatory impact per site was achieved by intersecting multiple independently curated annotation tracks (H3K4Me, H3K27Ac, DNase Clusters, Transcription Factor ChIP-seq). Given the independence between tracks, positions that re-occur in more than one track carry increased likelihood of regulatory function, giving a comprehensive overview of the regulatory DNA of the identified variants. For the key genes with $\geq 2$ NCCMs per 100 -kbp sequence, we observed that $85 \%$ of the NCCMs had alterations either in putative transcription factor binding sites (TFBS) (curated as well as Txn Factor ChIP validated annotations), ORegAnno elements (curated regulatory annotations), and/or other functional annotations such as DNase I hypersensitivity and/or DNA methylation in brain tissue. 


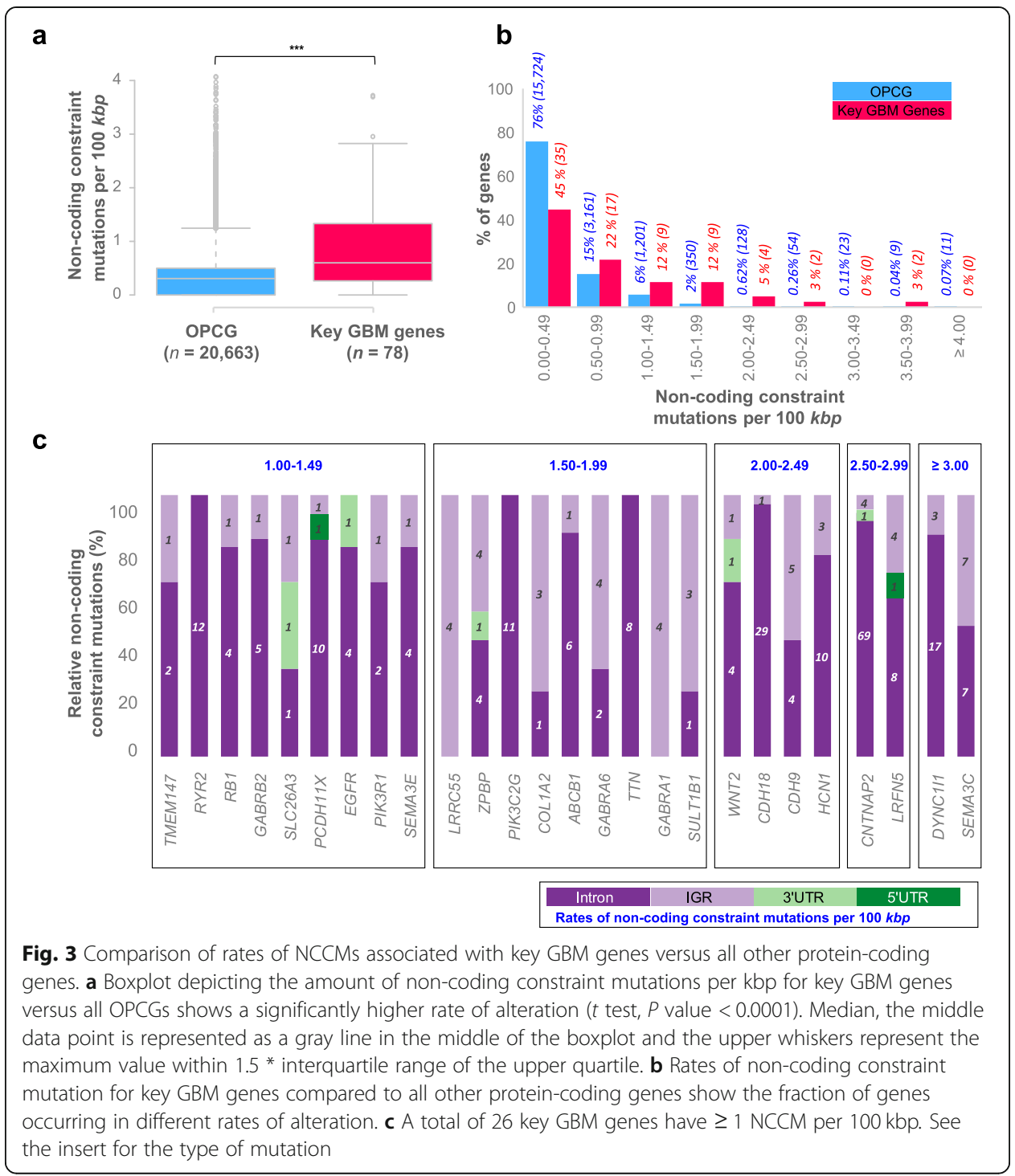

For semaphorin 3C, SEMA3C, a gene overexpressed in glioma and employed by glioma stem cells to promote tumorigenicity [38], we observe 14 NCCMs (in 11 patients; one or more $\mathrm{NCCM} /$ patient) with an equal distribution of intergenic and intronic NCCMs (7 each) (Fig. 4a, Additional file 6: Table S5). In addition, four of these NCCMs, number 9, 10,13, and 14, were present in regions predicted to bind transcription factors (TF) and are thus potentially of high biological relevance. Since TFs recognize and bind to specific genomic DNA, we decided to investigate if any of our above regulatory mutations were likely to perturb the binding affinity using the tool "TRanscription Factor Affinity Prediction" (sTRAP module) for detecting differences in binding between two sequences [39]. NCCM9 lay in the promoter region of the SEMA3C gene and overlapped with a FOXA1 binding site. The sTRAP analysis revealed that the region surrounding NCCM9 is predicted to be rich in TF binding sites and that there are differences in the binding affinity between the wild-type and the mutated sequence for several TFs (e.g., ZNF354C, FOXA2, EN1, RUNX1), and with the FOXA1 factor displaying a total lack of predicted binding in the mutated sequence 
a

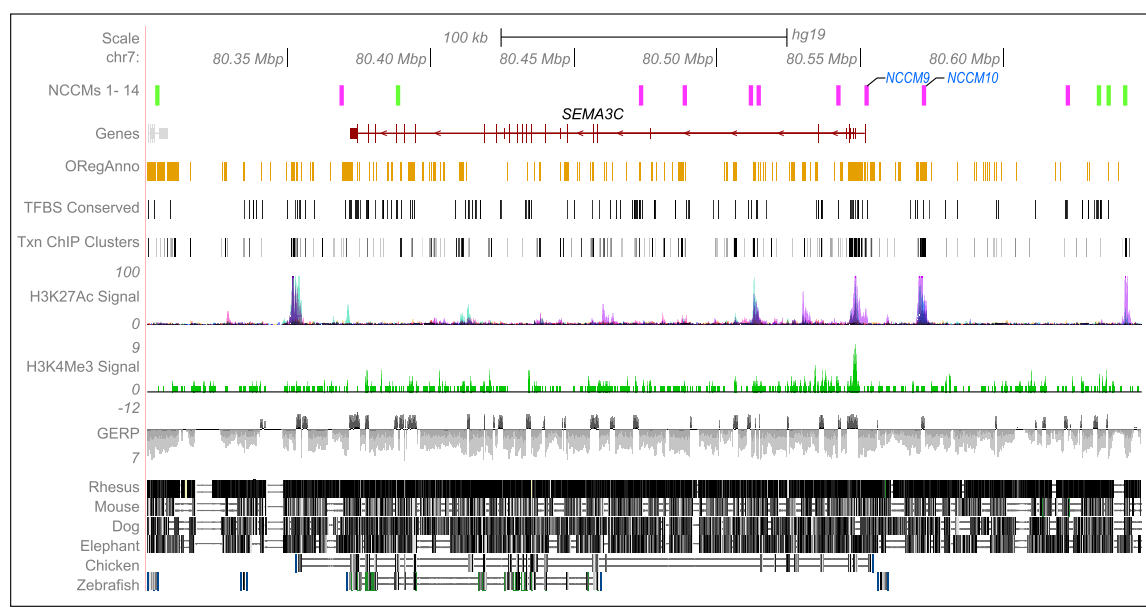

b

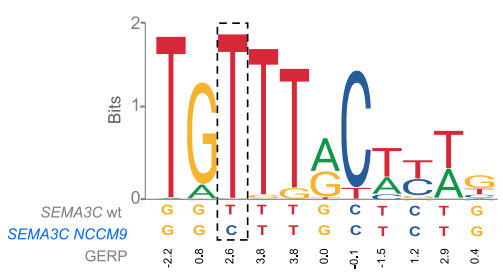

C

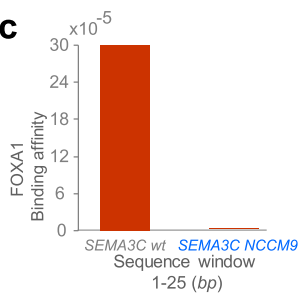

d

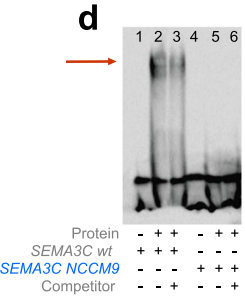

$\mathbf{e}$

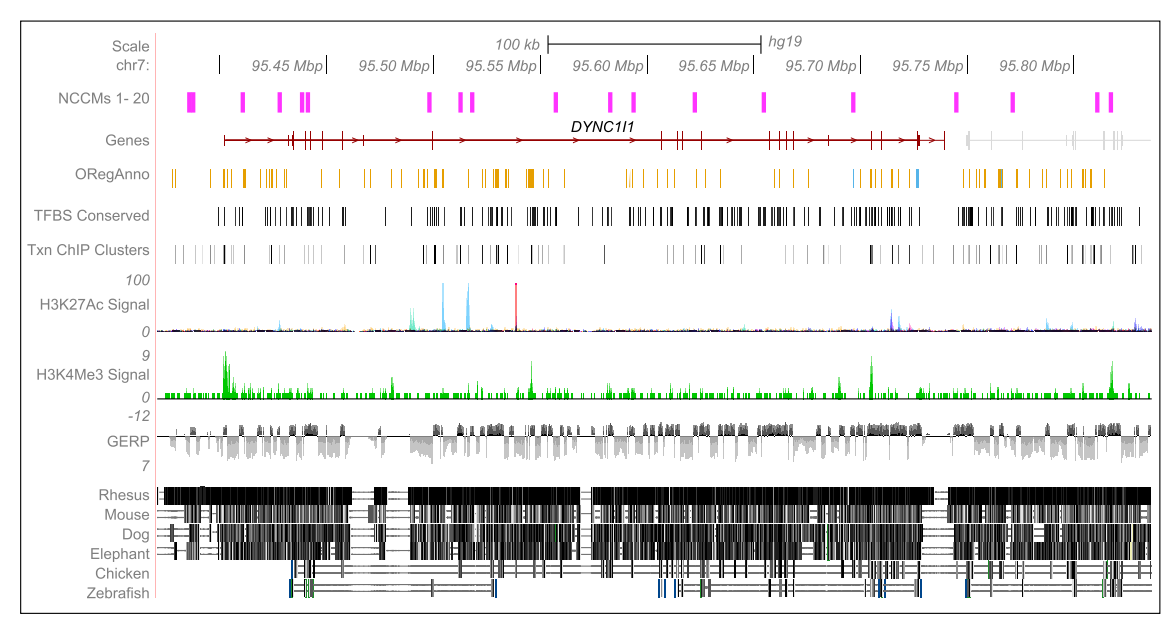

Fig. 4 UCSC genome browser view of SEMA3C and DYNC111, key GBM genes with the highest rates of noncoding constraint mutations. For each gene, the NCCM track shows the mutations, color-coded by VAF scores (green VAF 1-10\%; fuchsia VAF 11-45\%). a For SEMA3C, the 14 NCCMs seen both in introns and in the flanking intergenic regions lie in regions of the genome that are well conserved across mammals and 13 of 14 are associated with at least one additional regulatory annotation. $\mathbf{b}$ The sequence logo (MA0148.1) of the FOXA1 TFBS shows that the SEMA3C NCCM9 mutation affects a highly conserved nucleotide that could abate the binding in the mutated site compared to the wild-type. c The affinity profiles, for the same mutated sequence, shows a decreased affinity for the FOXA1 transcription factor, in the mutated compared with the wild-type sequence. $\mathbf{d}$ Electrophoretic mobility shift assay of DNA protein binding for SEMA3C wt and SEMA3C NCCM9. Purified nuclear protein from GBM cell line U3065MG was tested for DNA binding to either the predicted SEMA3C wt region dsDNA (lanes 2-3) or the corresponding SEMA3C NCCM9 region dsDNA (lanes 5-6). Unlabeled dsDNA for each region was used as competitor. The total lack of shift in lanes 5-6 confirms abolition of DNA binding capacity as a consequence of the mutation. e In DYNC1/1, the majority of the 20 NCCMs are seen in the intronic regions of the gene and in regions with mammalian conservation. In addition, regulatory annotations associated with promoters and conserved TFBS are also seen 
(Fig. 4b and c, Additional file 7: Table S6). For comparison, we performed sTRAP analysis for both the wild-type and mutated sequence for position 6 in the FOXA1 binding matrix. The position is not conserved in the matrix and did not display any differences in the binding affinities. DNA-protein binding activity for NCCM9 was further confirmed by an electrophoretic mobility shift assay (EMSA) (Supplementary Table 5, Fig. 4d). Biotin-labeled DNA probes containing the predicted DNA binding site, either for the wild-type (wt) or for the mutant sequence (NCCM9), were used, with unlabeled DNA probe as control to determine binding specificity. No binding was observed for the mutant sequence, indicating that NCCM9 leads to loss of DNA binding in this region. NCCM9 also had a VAF of $27 \%$, indicating that it likely was an early event in the evolution of the tumor. Nine other NCCMs in SEMA3C also have at least one more functional annotation together with evolutionary constraint (Additional file 6: Table S5).

The DYNC1I1 (dynein, cytoplasmic 1, intermediate chain 1) gene is known to be downregulated in glioma, and its low expression is correlated with poorer patient survival [40]. We found 20 NCCMs for DYNC1I1 (17 intronic and three intergenic, across 16 patients, Fig. 4e, Additional file 8: Table S7). The activating epigenetic marker H3K27me3 (tri-methylation on lysine 4 of histone) is often found in promoter regions and is closely associated with transcriptionally active genes. Nine of the DYNC1I1 variants have annotations for an H3K4me3 mark, and three of the intronic NCCMs, number 4, 10, and 15, intersected DNase I hypersensitive sites (DHSs), indicative of regions of accessible chromatin. Four NCCMs were found to lie in the transcription factor binding site (TFBS) of the HAND1-E47 heterodimer (Additional file 5: Table S4), and the 3'-UTR motif of this co-regulated gene cluster has been reported to bind to highly conserved DNA regions in lung adenocarcinomas [41]. Most of the NCCMs' positions overlap with brain methylation signals (as discerned from the UCSF brain methylation track [42]; Additional file 8: Table S7). DYNC111 NCCM15 is situated in a regulatory region that has a possible enhancer role. The sTRAP analysis for the above three NCCMs indicated significant differences between the wild-type and the mutated sequences in their binding affinity to several TFs including multiple members of the GATA family (Additional file 7: Table S6).

LRFN5, a fibronectin type III domain-containing protein that mediates cell-cell adhesion in a $\mathrm{Ca}^{2+}$ independent manner, had 14 NCCMs, all with VAF > 30\% (across 13 patients, Additional file 5: Table S4). Eight of these mutations were found in introns, four in intergenic and one alteration each overlapped the $5^{\prime}$-UTR and lincRNA regions. NCCM5, a $5^{\prime}$-UTR mutation, is conserved across all 33 vertebrates and overlap with the oncogenic lncRNA CTD-2298 J14.2 [43] and TFBS for MEF2A. Three NCCMs, 5, 6 and 13, are found in large conserved regions of the genome and have TFBS predictions within $20 \mathrm{bp}$ of the variant. sTRAP analysis also showed a significant shift in the binding affinity between the mutated and wild-type sequences for several TFs (Additional file 7: Table S6).

CNTNAP2 (Contactin-associated protein-like 2), a member of the neurexin family, has been proposed as a tumor suppressor in glioma [44], and in our cohort, CNTNAP2 showed 74 NCCMs with 29 patients having one or more variants. The majority of these NCCMs were found in introns, and several of the annotations relate to epigenetic regulation in the brain and other tissues (Additional file 5: Table S4). 
Identification of additional genes with enrichment of NCCMs

To uncover novel genes with putative roles in GBM, we focused on the tail of the distribution of the OPCGs which showed that 1776 genes had $\geq 1.0$ non-coding constraint mutations per 100-kbp sequence (Fig. $3 \mathrm{~b}$ ) and that 43 showed $\geq 3.0$ non-coding constraint mutations per $100-\mathrm{kbp}$ sequence. Of the 43 genes that satisfied this criterion (Fig. 5), a total of 15 have previously been reported to have altered expression patterns in GBM, while 28 genes have hitherto no known roles in the disease. Eleven of the 43 genes have $\geq 4.0$ NCCMs per 100-kbp sequence (Fig. 5), and mutations for the top four genes DLX5, DLX6, FOXA1, and ISL1 are distributed over promoters, UTRs, and multiple TFBS regions (Additional file 9: Table S8) and are expected to affect many transcription factor-binding motifs. We also note that approximately one third of these OPCGs with $\geq 4.0 \mathrm{NCCMs} / 100 \mathrm{kbp}$ occur in clusters, one on chromosome 6 (OPN5, PTCHD4, TFAP2D, TFAP2B and PKHD1), two clusters on chromosome 7 (DLX5, $D L X 6)$ and (CAV1, CAV2, MET), and one cluster on chromosome 14 (SLC25A21, MIPOL1, FOXA1, TTC6).

The homeobox gene DLX5, Distal-Less Homeobox 5, has been shown to affect glioma cell motility via the PAX6/DLX5-WNT5A axis [45]. DLX6 is a paralog of DLX5, and the two genes are located in a tail-to-tail configuration on chromosome 7. Also, there is a long non-coding RNA, DLX6-AS1, the expression of which has been reported to correlate with worse patient outcome in GBM [46]. Together, the queried territory for $D L X 5 / 6$ showed 15 NCCMs (across 10 patients), most of which are intergenic (Fig. 6a, Additional file 10: Table S9). NCCMs 9 and 10 are located in highly conserved regions and overlap TFBS annotations for SP1 and ZIC1. The NCCMs corresponding to these positions show significant differences in their binding affinities when compared with their corresponding wild-type sequences (Additional file 7: Table S6). The position for NCCM7 is highly conserved and likely located in the promoter region of the long non-coding RNA DLX6-AS1 (Additional file 10 Table S9).

The genes SLC25A21, MIPOL1, FOXA1, and TTC6 lie in close proximity on chromosome 14 (37.1-38.6 Mbp), and therefore, the identified 56 NCCMs (across 22 patients)

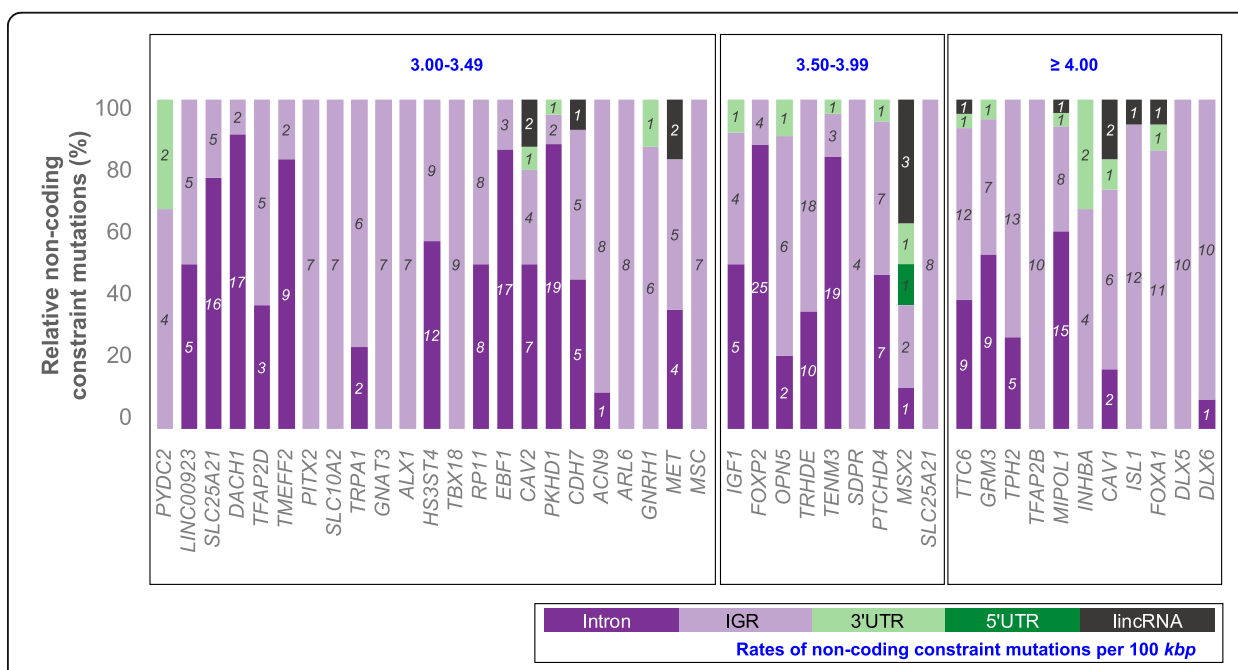

Fig. 5 Additional genes with NCCMs enrichment. A total of 43 genes outside the key GBM gene list had $\geq$ 3 NCCMs per kbp (see the insert for the type of mutation) 


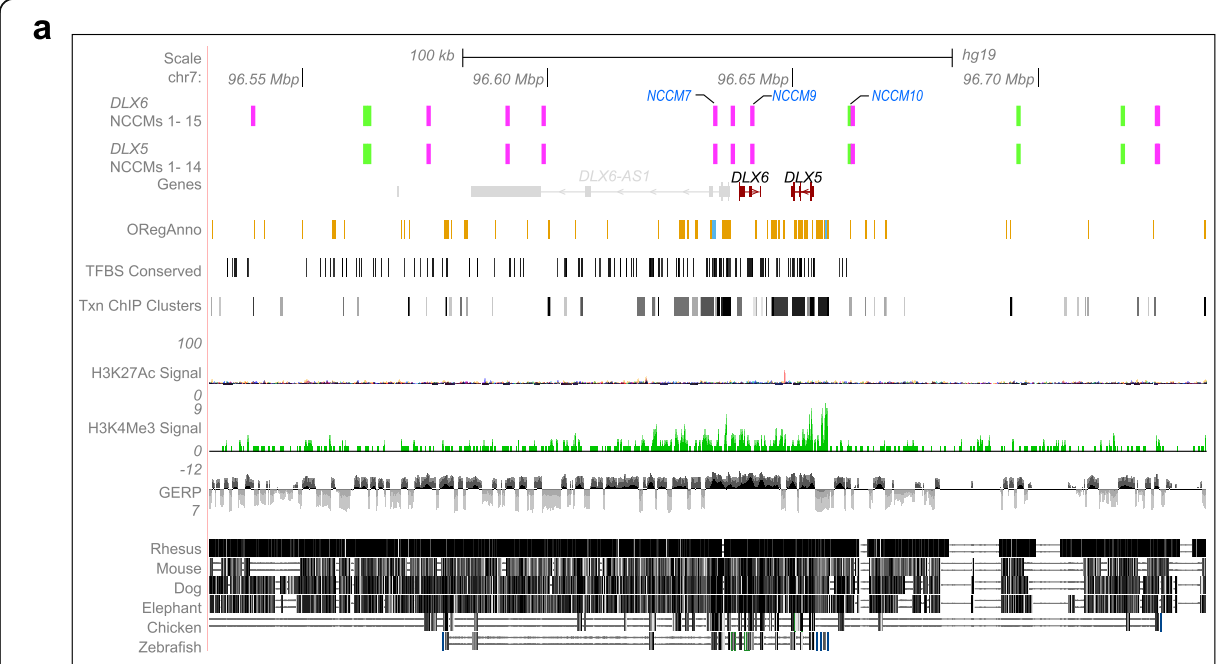

b

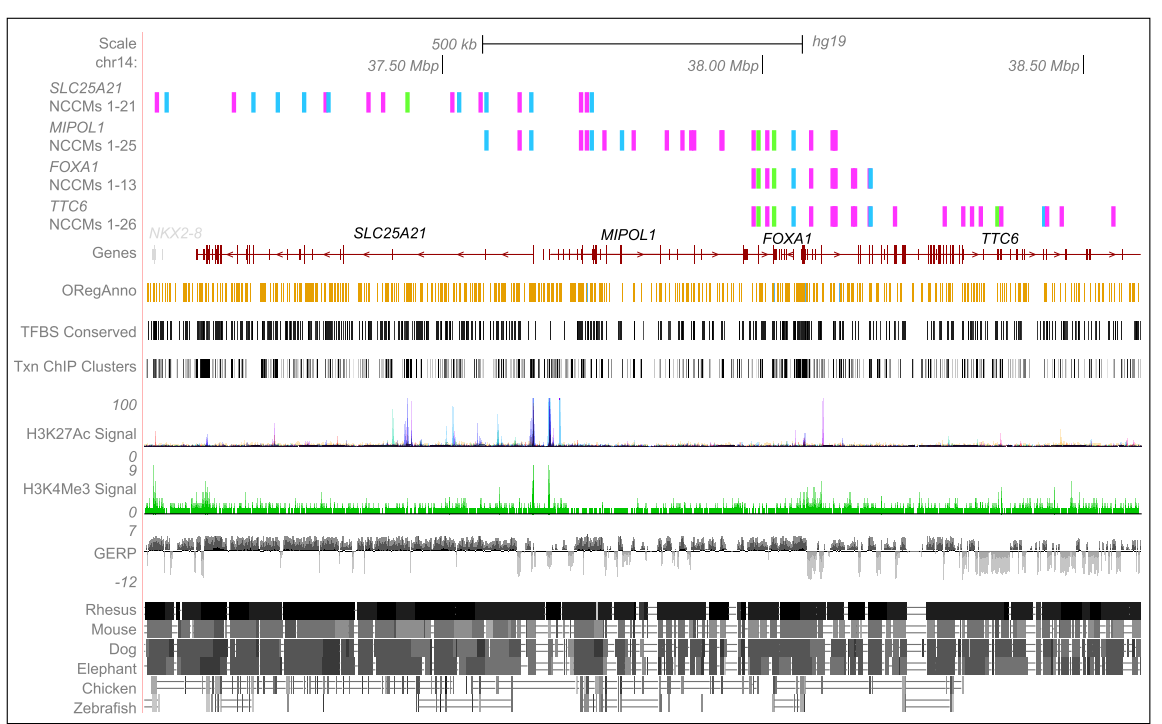

Fig. 6 UCSC genome browser view of DLX5 and DLX6 and SLC25A21, MIPOL1, FOXA1, and TTC6 NCCMs clusters. For each gene, the NCCM track shows mutations, color-coded by VAF scores (electric green VAF 110\%; fuchsia pink VAF 11-45\%; blue VAF > 46\%). a Variants associated with the DLX genes overlap strong methylation patterns and are found in regions of mammalian conservation. b For SLC25A21, MIPOL1, FOXA1, and $\Pi C 6$, their associated NCCMs are found in regions with regulatory potential, including promoters and TFBS

largely overlap between the four genes although SLC25A21 had 14 and TTC6 had 13 unique NCCMs, respectively (Fig. 6b, Additional file 2: Table S10). Some genes in this locus have been implicated in glioma; the SLC25A21gene is downregulated in GBM [47], and increased FOXA1 transcription has been reported to promote glioma proliferation [48]. MIPOL1, mirror-image polydactyly 1, is vital for CNS development and is also known to have a tumor suppressor role in nasopharyngeal cancer [49]. The tetratricopeptide repeat domain 6, TTC6, has been shown to play a role in breast cancer, and in prostate cancer, there is evidence for a TTC6-MIPOL1 fusion [50]. The annotations associated with the NCCMs that span the four genes reveal that most have good 
conservation as well as multiple TFBS annotations (Additional file 11: Table S10). Here again, sTRAP analysis for the mutated sequences versus the wild-type showed significant differences in their binding affinity (Additional file 7: Table S6).

\section{Identification of mutational signatures}

Different mutational processes leave distinctive "stamps" of somatic alterations in cancer cells; to discern these patterns, the tool "MUTation AnaLyIS toolKit" (Mutalisk [51]) was utilized. Two signatures, COSMIC 1, the so-called aging signature, and COSMIC 5, are seen as the dominant profiles across our samples (Additional file 1: Figure S5a) which is also in accord with previously detected signatures in GBM [52]. While present in many cancers, there is no clear known etiology for COSMIC 5. In addition to COSMIC1 and COSMIC5, COSMIC 11 has been reported in patients treated with the alkylating agent TMZ [52]. COSMIC11 was the overriding signature (86\%), in our hyper-mutated recurrent tumor sample T-3151 (Additional file 1: Figure S5a). T-3151 is also the only sample to show a robust signal (14\%) for COSMIC 14 , a signature that is known to generate a very high number of somatic alterations [52]. Among the three other recurrent tumors in the cohort (T-3179, T-3233, and T-3243), which were also treated with TMZ, none exhibit COSMIC 14, and in T-3179, a minor contribution of COSMIC 11 was observed.

Interestingly, when the variants were split into coding and non-coding alterations, the former showed predominance for the aging signature, whereas the non-coding variants showed near equal distribution between the COSMIC 1 and COSMIC 5 signatures (Additional file 1: Figure S5b). Furthermore, when the mutations were divided based on being either non-constraint or constraint, we observed a significant difference in the signature distribution between the two categories (Additional file 1: Figure S5c), with COSMIC 5 dominating in the constraint changes. This dominance is also seen when the constraint dataset is parsed into non-coding changes (Additional file 1: Figure S5d).

\section{Discussion}

Alterations in the non-coding regulatory regions of GBM largely remain unexplored. The principal goal for this study was to characterize, in a genome-wide context, the non-coding mutations that arise in GBM tumors. Here, we provide novel insights into the somatic changes affecting the regulatory landscape of GBM. Despite a relatively small cohort, our 12 significantly mutated genes (SMGs) and frequently mutated genes (FMGs) were all found among the top 20 most frequently mutated protein-coding genes in the TCGA GBM cohort. Similarly, in comparison with the same dataset, $>95 \%$ of the amplified and deleted genes are also found to be altered in our cohort. Thus, being able to mirror the SCNA landscape and the chief protein-coding mutations, as well as the mutational signatures previously found in the large TCGA cohort, lend credence to our cohort and the findings derived from it.

Mutations within the functional, non-coding regions of the genome can alter gene expression, splicing, expression of non-coding transcripts, and the epigenetic state [33]. As of today, no studies have focused on the non-coding constraint regulatory elements of the GBM genome, and thus, the impact of non-coding mutations remains largely 
uncharacterized. The main challenge has been to distinguish between passenger and driver mutations in the non-coding regions, where 5-10\% [53] of bases have been estimated to be functional. Evolutionary conservation provides an empirical way of identifying which specific positions are important for genome function [54]. We compared 78 "key GBM genes," identified based on frequent coding mutations, with all other protein-coding genes in the genome. Examining the sequence within and around each gene $( \pm 100 \mathrm{kbp})$, and using a cut-off of GERP $>2$ as a definition of a constrained site, we identified a highly significant enrichment of NCCMs in the neighborhood of the 78 key GBM genes with 26 genes being enriched for NCCMs (>1 NCCMs/100 kbp). The key genes with the highest frequency of NCCMs were SEMA3C, DYNC1I1, CNTNAP2, and LRFN5.

While the NCCM frequencies in key GBM genes were skewed to the right, compared to all other protein-coding genes, the latter category still contained genes with high NCCM frequency. In total, 1776 genes had a frequency of $>1$ NCCMs/ $100 \mathrm{kbp}$, and we further studied the 43 genes with a frequency of $>3 \mathrm{NCCMs} /$ $100 \mathrm{kbp}$. Overall, the distribution of NCCMs to their location category varied, with some genes (typically large ones) showing mostly intronic NCCMs, while others contained mostly intergenic variants. In an attempt to assign candidate functions to the NCCMs, we used publicly available sources of genome annotations, in addition to the evolutionary constraint, and found that most NCCMs had functional annotations, suggesting that they may indeed be driver mutations. We also noted that $91 \%$ of NCCMs in the top genes (26 key GBM genes and 43 from all other protein-coding genes) had a VAF of $\geq 10 \%$, again supporting their ability to affect the tumor initiation and/or progression.

When assessing the potential biological importance of the analyzed variants, SEMA3C stood out, jointly due to its large number of NCCMs and the existing literature suggesting that overexpression of $S E M A 3 C$ is linked to poor prognosis in several cancer types including prostate cancer [55] and GBM [56]. In the topological associated domain (TAD; chr7:80.4-81.1 Mbp) that contains the SEMA3C gene, we found 14 NCCMs. The SEMA3C gene has been shown to be regulated by several TFs, including FOXA1, GATA2, and GATA6, as well as by the androgen receptor. From our sTRAP analysis, NCCM9, in particular, clearly showed that the mutation would disrupt FOXA1 binding. This was experimentally validated in an electrophoretic mobility shift assay where the DNA-protein binding was reduced for the NCCM9 template. The DNA binding to the wild-type allele could not be competed out by an excess of unlabeled probe with the same sequence. This is a known issue for some DNA binding factors [57] but can potentially be overcome by using Multiplexed Competitor Electrophoretic Mobility Shift Assay (MC-EMSA) where the DNA binding to one probe is competed with a cocktail of probes with slightly different sequences [57]. Intriguingly, we also note that $50 \%$ of females have a SEMA3C mutation while only $25 \%$ of males do, possibly suggesting that the relative lack of the androgen receptor in females means that to obtain the same effect, the mutations need to occur directly in the SEMA3C gene to cause overexpression.

Additionally, multiple variants occur in binding sites for the GATA family of transcription factors. Given that GATA factors are known to coordinate cell survival, 
cellular maturation, and proliferation arrest [58], this family of genes has been anticipated to have a role in human cancers [59]. Variants for SEMA3C, DYNC1I1, and CDH18 lie in conserved TFBS of the GATA family. GATA2 has been directly implicated in promotion of glioma through the EGFR/ERK/Elk-1 pathway [60], further indicating its potential to forward tumor development in GBM.

The discovery of non-coding driver mutations in GBM is still an emerging field and has the potential to become an integral part of clinical studies and precision medicine in the future. Here, we suggest that evolutionary constraint, combined with other genomic annotation information, could provide a useful approach to identify novel candidate GBM mutations. We hope that the findings from this study will provide a solid foundation for functional validation of novel non-coding, evolutionarily constrained candidate mutations in GBM.

\section{Conclusions}

The results presented herein suggest that non-coding constraint mutations could play an essential role in GBM, underscoring the need to connect non-coding genomic variation to biological function and disease pathology.

\section{Materials and methods}

\section{Patient cohort and ethical consent}

The GBM patients for the study were included in the "Uppsala-Umeå Comprehensive Cancer Consortium" (U-CAN) biobank (www.u-can.uu.se). U-CAN is a resource for longitudinal sampling of tumor tissues, blood, and associated clinical data from cancer patients, all of which are collected with their informed consent [61]. The study was approved by the Ethical Review Board of Uppsala, Sweden (Dnr 2007/353 and addenda 2013-10-28, 2016-12-29) (Uppsala Biobank no: 827-2014-087, U-CAN: 2014004), and all work involving human tissue samples were conducted in accordance with the Declaration of Helsinki. Matched tumor-normal specimens for sequencing were selected based on the histopathological annotation of the tumor tissues by a neuropathologist. Samples assessed as having at least $40 \%$ tumor cells were selected for WGS. The cohort was designated as SweGBM-1, and the individual samples were labeled according to their Human Glioma Cell Culture identity (HGCC [62]) with " $\mathrm{T}$ " and " $\mathrm{N}$ " prefixes used to denote tumor tissue and normal blood sample respectively. Based on specimen availability that satisfied the quality thresholds for the histopathological criterion stated above, samples from 31 male and 8 female patients, with age of diagnosis ranging from 38 to 83 years (median 65 years), were selected for the study resulting in a cohort size of $n=39$ (Fig. 1).

\section{DNA preparation, library construction, and WGS}

Tumor sections (3-18 sections, contingent on the extent of necrosis) of $10 \mu \mathrm{M}$ were used to prepare tumor DNA using the "AllPrep DNA/RNA/miRNA Universal Kit" (Cat. no 80224, Qiagen, Hilden, Germany) in line with the manufacturer's protocol. Blood DNA was using the Blood DNA kit (Cat. No 51104, Qiagen, Hilden, Germany). Tumor and normal DNA was then submitted to NGI Uppsala SNP/SEQ facility at SciLifeLab. All samples that passed the threshold values for 
library preparation were sequenced using the Illumina HiSeqX and True Seq PCR-free methods. Minimum target depths of coverage of $60 \times$ for the tumor and $30 \times$ for the normal were set for the sequencing, and the above protocol yielded paired-end reads of 150-bp read length for each of the matched normal and tumors in the cohort.

\section{Sequence data alignment}

To align the raw WGS reads to the reference assembly hg19, BWA v0.7.15 [63] with default options was used. The resulting binary alignment map (BAM) files were then refined following Genome Analysis Toolkit's (GATK [64]) recommended best practices available through the website https://software.broadinstitute.org/gatk/bestpractices/. These included removal of optical duplicates with Picard's (http://broad institute.github.io/picard/) utility MarkDuplicates, correction of local realignment around indels via GATK's Indel-Realigner module, and recalibrating and reducing machine-read error induced noise from individual base quality score with GATK's base quality score recalibration (BQSR) module (Additional file 1: Figure S6).

\section{TCGA dataset used for comparative analysis}

The TCGA dataset, "Glioblastoma Multiforme (TCGA, Provisional)," downloaded from cBioPortal was used for all comparative analyses.

\section{Somatic Copy Number Alteration (SCNA) calling and comparison with TCGA SCNA data}

Somatic copy number aberrations present in tumor samples were determined with ascatNGS (Additional file 1: Figure S6 [18]). For a given sample, matched refined Tand N- BAM files were provided as input data to the tool and executed with default options. Resulting "seg" output files were then used for comparison with the TCGA SCNA dataset. To assess if the SweGBM-1 copy number profiles had alterations that matched with the TCGA, GISTIC defined recurrent SCNAs, data for the latter was first downloaded from the cBioPortal, after which a custom script was run to find regions that overlapped in the two cohorts.

\section{Somatic variant calling and filtration}

The tumor $(\mathrm{T})$ - and normal (N)- BAM files were also used as input for the somatic mutation calling tools of MuTect2 v3.7-0 [21] and Strelka v1.0.15 [22]. Each of these tools output a variant calling format (VCF) file that contains somatic point and indel mutations present per matched $\mathrm{T} / \mathrm{N}$ sample. To eliminate potential sequencing and germline artifacts and other sources of false-positive calls that may be present in the VCFs, a series of filtration steps were performed. Firstly, calls that were marked as germline SNPs in at least two samples in a "Panel of Normals" (PoN) dataset were filtered out. The PoN dataset was built from filtered SNPs/germline variants called by the GATK's HaplotypeCaller across the normal samples of the SweGBM-1 cohort. Another round of filtering involved removal of germline calls that were present in the publicly available databases of dbSNP v150 [65] and SweGen Variant Frequency Dataset [66]. Since there is a possibility that in these two databases some somatic variants are erroneously marked as 
germline variants, calls present in either resources which also overlapped with somatic variants present in the COSMIC database [26] were "whitelisted," i.e., not filtered out. To further minimize the rate of false-positive calls, the list of filtered calls generated with MuTect2 and Strelka was intersected. Only the somatic point mutations (SPM) and somatic indel mutations (SIM) that were in consensus between the callers were used as the final call set for all downstream analysis.

\section{Classification of SweGBM-1 SPM and SIM calls and identification of significantly mutated genes}

To functionally classify variants into coding and non-coding categories, the SPM and SIM calls were annotated by the tool Oncotator [23]. To discover genes that may contain putative coding driver mutations, two methods were adopted: first, a statistical approach using the tool MutSigCV [24] with default options was run to get significantly mutated genes (SMGs). The counts here were then augmented with a frequency-based approach, wherein genes that were mutated in $\geq 4$ tumor samples (approximately 10\% of samples) were tallied to get a list of frequently mutated genes (FMGs).

\section{Visualization of the somatic alterations}

To visualize alterations in the SMGs and FMGs, oncoprints and lollipop plots were generated using tools available on the web-based utility cBioPortal [27].

\section{TERT promoter mutation analysis}

The TERT promoter locus encompassing known hotspot mutations on chromosome 5 at positions 1,295,228 and 1,295,250 was examined for alterations in the MuTect2Strelka concordant dataset for all of the samples.

\section{Defining a key GBM gene set for the investigation of non-coding variants with regulatory potential}

Alterations in key genes, especially SMGs, are known to be associated with GBM tumor initiation and/or progression. To investigate if non-coding regulatory variants of these key GBM genes might contribute to the GBM phenotype, we compiled a set of genes by pooling together the list of the SMGs $(n=71$ [27] $)$ from the TCGA-GBM cohort and SMGs/FMGs $(n=12)$ from the SweGBM-1, resulting in a total of $n=78$ genes (Additional file 4: Table S3).

Non-coding variants associated with the above-described key genes were then examined in greater detail. GERP RS scores, a measure of sequence conservation, were collated for every SPM and SIM across the cohort, based on the data from the UCSC GERP RS conservation track [16]. Non-coding variants, whose position had a GERP RS score $\geq 2$ and which were located within $3^{\prime}$ - or $5^{\prime}$-UTRs, introns, or \pm 100 -kbp intergenic flanking regions, were extracted. These variants, referred to hereafter as non-coding constrained mutations, or NCCMs, were subsequently annotated with regulatory annotations downloaded from either the UCSC genome browser or ENCODE portal, or both. These include, among others, information from tracks pertaining to transcription factor binding sites (TFBS), methylation 
and histone modification markers (H3K4Me, H3K27Ac), regulatory markers (ORegAnno), transcription start sites, enhancer information, and chromatin immunoprecipitation assay data of DNA regions where TFs/proteins bind (Txn Factor ChIP) (Additional file 1: Figure S7).

\section{Statistical tests for enrichment analysis}

The rate of NCCMs around key genes was compared with the same class of variants associated with all other protein-coding genes (OPCG). A $t$ test was performed using the R statistical framework (R Foundation for Statistical Computing, Vienna, Austria. http://www.R-project.org/).

\section{Transcription factor binding affinity prediction}

The sTRAP module from the TRAP tools (http://trap.molgen.mpg.de/cgi-bin/trap_form. cgi) was used to predict if regulatory sequences associated with NCCMs of key GBM genes and OPCG could alter transcription factor binding affinity to DNA. For every NCCM, 41bp sequences were analyzed with the wild-type or the mutant allele centered. The matrices for the analysis were set to the JASPAR database, and for the background model "human promoter," option was selected. The JASPAR database (http://jaspar.genereg.net) was used to obtain information about TF binding matrices and motifs of interest.

\section{EMSA (electrophoretic mobility shift assay)}

For the NCCM9 and wild-type sequence in the SEMA3C promoter, $5^{\prime}$ biotin-labeled and unlabeled forward strand DNA oligos and their reverse complementary unlabeled strand (HPLC-purified, purchased from IDT (Integrated DNA Technologies) were as follows:

wt_F:GCAACAGTGGTTTGCTCTGGAGAGGAAA

wt_R:TTTCCTCTCCAGAGCAAACCACTGTTGC

NCCM9_ F:GCAACAGTGGCTTGCTCTGGAGAGGAAA

NCCM9_R:TTTCCTCTCCAGAGCAAGCCACTGTTGC

Oligos were first annealed in equimolar amounts in $1 \times$ annealing buffer $(50 \mathrm{mM}$ $\mathrm{NaCl}, 10 \mathrm{mM}$ Tris- $\mathrm{HCl}, 10 \mathrm{mM} \mathrm{MgCl} 2,100 \mu \mathrm{g} / \mathrm{ml} \mathrm{BSA}, \mathrm{pH} 7.9$ at $25^{\circ} \mathrm{C}$ ) in a thermo cycler by heating to $95^{\circ} \mathrm{C}$ for $5 \mathrm{~min}$ and gradual cooling at $1{ }^{\circ} \mathrm{C} / \mathrm{min}$ to $4{ }^{\circ} \mathrm{C}$. Nuclear protein was extracted from patient-derived glioblastoma cells U3065MG [62] using the NucBuster ${ }^{\text {ru }}$ Protein Extraction Kit (Millipore). The subsequent binding reaction of the dsDNA with nuclear protein extract was processed using LightShift ${ }^{\text {tw }}$ Chemiluminescent EMSA Kit (Thermo Scientific) as per the manufacturer's protocol and incubated on ice for $40 \mathrm{~min}$ for the binding reaction. Following this, $5 \mu \mathrm{l}$ of loading buffer was mixed with each binding reaction and a total of $20 \mu \mathrm{l}$ per reaction was then loaded per well onto a Bio-Rad Criterion gel (Bio-Rad) and electrophoresed for $90 \mathrm{~min}$ at $200 \mathrm{~V}$. The gel was transferred to a GeneScreen Plus nylon hyrbidization transfer membrane (PerkinElmer) for $1 \mathrm{~h}$ at $45 \mathrm{~V}$ followed by UV crosslinking for 15 min with the membrane facing down on a transilluminator and additional 1 more minute with the membrane turned over. The membrane was developed using the Chemiluminescent Nucleic Acid Detection Module Kit (Thermo Scientific) and visualized on the Bio-Rad CCD camera (Bio-Rad). 


\section{Mutational signatures discovery}

The mutational signature detection for the cohort was performed with the online tool Mutalisk [51]. VCF files for the cohort were used as inputs, and the maximum likelihood with linear regression options was turned on. A specific cancer type was not selected for.

\section{Supplementary information}

Supplementary information accompanies this paper at https://doi.org/10.1186/s13059-020-02035-x.

Additional file 1: Figure S1. Somatic Copy Number Alteration (SCNA) in SweGBM-1 matches observations in the TCGA-GBM dataset. Figure S2. Distribution of Coding and Non-Coding variants per sample for the SweGBM-1 Cohort. Figure S3. TERT $T^{p}$ mutational profiles for the SweGBM-1 cohort. Missense mutations at two positions in the TERT promoter observed previously in GBM datasets are seen in most samples. Figure S4. Boxplot of the rates of constraint bases in the internal and flanking regions of key GBM and all OPCG. Figure S5. Mutalisk algorithm identifies two major mutational signatures, Cosmic 1 and Cosmic 5 across samples. Figure S6. Workflow for variant and copy number detection in matched tumor-normal samples. Figure S7. Workflow for annotation of noncoding constraint mutations.

Additional file 2: Table S1. TCGA top 20 GBM genes that overlap with SweGBM-1 cohort.

Additional file 3: Table S2. Intersection of alterations in SweGBM-1 FMGs with TCGA-GBM dataset and with pancancer databases of COSMIC, CBioPortal and CancerHotspots.

Additional file 4: Table S3. List of Key GBM genes $(n=78)$, pooled from SweGBM-1 FMGs and TCGA-GBM SMGs.

Additional file 5: Table S4. Annotation of the NCCMs associated with the key GBM genes based on data from the ENCODE portal and the UCSC Browser databases.

Additional file 6: Table S5. Annotation of the NCCMs associated with the SEMA3C gene based on data from the ENCODE portal and the UCSC Browser databases.

Additional file 7: Table S6. Regulatory analysis of select Key GBM and OPCG NCCMs are shown here. The top five ranks of the most substantial difference of affinity between wildtype and mutated sequences are listed.

Additional file 8: Table S7. Annotation of the NCCMs associated with the DYNC1/1 gene based on data from the ENCODE portal and the UCSC Browser databases.

Additional file 9: Table S8. Annotation of select genes outside the Key GBM genes', associated NCCMs with data from the ENCODE portal and UCSC browser databases.

Additional file 10: Table S9. Annotation of the NCCMS associated with the DLX5 and DLX6 genes based on data from the ENCODE portal and the UCSC Browser databases.

Additional file 11: Table S10. Annotation of the NCCMs associated with the SLC25A21, MIPOL1, FOXA1, and TTC6 genes based on data from the ENCODE portal and the UCSC Browser databases.

Additional file 12. Review history.

\section{Acknowledgements}

The authors thank Dr. I. Alafuzoff, Department of Immunology, Genetics and Pathology, Uppsala University, for annotation of GBM patient samples; Dr. M. Mayrhofer at the National Bioinformatics Infrastructure Sweden and SciLifeLab Swedish Bioinformatics Advisory Program for bioinformatics advice; and Åsa Karlsson for technical assistance. The authors acknowledge support from the National Genomics Infrastructure in Uppsala funded by Science for Life Laboratory, the Knut and Alice Wallenberg Foundation and the Swedish Research Council, and SNIC/Uppsala Multidisciplinary Center for Advanced Computational Science for assistance with massively parallel sequencing and access to the UPPMAX computational infrastructure.

\section{Peer review information}

Yixin Yao was the primary editor on this article and managed its editorial process and peer review in collaboration with the rest of the editorial team.

\section{Review history}

Review history is available as Additional file 12.

\section{Authors' contributions}

Conception and design: KLT, KFN. Development of methodology: SS, MP, KLT. Acquisition of data: LH, AR, ES. Analysis and interpretation of data: SS, AR, LH, MP, VM, ES, KLT, KFN. Writing, review, and/or revision of the manuscript: SS, AR, $L H, M P, V M, E S, K L T, K F N$. The author(s) read and approved the final manuscript.

\section{Authors' information}

Twitter handle: @ForsbergNilsson (Karin Forsberg-Nilsson).

\section{Funding}

This work was financially supported by grants from SciLifeLab, U-CAN, Swedish Research Council, Swedish Cancer Society, and Johan's Minne.

Open access funding provided by Uppsala University. 


\section{Availability of data and materials}

Somatic variation sequence data has been deposited at the European Genome-phenome Archive (EGA, https://www. ega-archive.org), which is hosted by the EBI and the CRG, under accession number EGAS00001004379 [67]. Additional processed data is available at https://doi.org/10.17044/nbis/g000010 together with information on how to submit data access requests. Tumor tissue samples may be requested from the U-CAN biobank (https://www.u-can.uu.se).

\section{Ethics approval and consent to participate}

The study was approved by the Ethical Review Board of Uppsala, Sweden Dnr 2007/353 and addenda 2013-10-28 and 2016-12-29, Uppsala Biobank no: 827-2014-087, U-CAN: 2014-004. The GBM patients for the study were included in the "Uppsala-Umeå Comprehensive Cancer Consortium" (U-CAN) biobank (www.u-can.uu.se), and samples were collected with their informed consent.

\section{Consent for publication}

Not applicable.

\section{Competing interests}

The authors declare that they have no competing interests.

\section{Author details}

'Department of Medical Biochemistry and Microbiology, Science for Life Laboratory, Uppsala University, SE-751 23 Uppsala, Sweden. ${ }^{2}$ Broad Institute, Cambridge, MA 02142, USA. ${ }^{3}$ Department of Immunology, Genetics and Pathology, Science for Life Laboratory, Uppsala University, SE-751 85 Uppsala, Sweden.

Received: 28 October 2019 Accepted: 30 April 2020

Published online: 09 June 2020

\section{References}

1. Sottoriva A, Spiteri I, Piccirillo SG, Touloumis A, Collins VP, Marioni JC, Curtis C, Watts C, Tavare S. Intratumor heterogeneity in human glioblastoma reflects cancer evolutionary dynamics. Proc Natl Acad Sci U S A. 2013; 110:4009-14.

2. Patel AP, Tirosh I, Trombetta JJ, Shalek AK, Gillespie SM, Wakimoto H, Cahill DP, Nahed BV, Curry WT, Martuza RL, et al. Single-cell RNA-seq highlights intratumoral heterogeneity in primary glioblastoma. Science. 2014;344:1396-401.

3. Stupp R, Mason WP, van den Bent MJ, Weller M, Fisher B, Taphoorn MJ, Belanger K, Brandes AA, Marosi C, Bogdahn U, et al. Radiotherapy plus concomitant and adjuvant temozolomide for glioblastoma. N Engl J Med. 2005;352:987-96.

4. Turkalp Z, Karamchandani J, Das S. IDH mutation in glioma: new insights and promises for the future. JAMA Neurol. 2014:71:1319-25.

5. $\quad$ Ohgaki H, Kleihues P. The definition of primary and secondary glioblastoma. Clin Cancer Res. 2013;19:764-72.

6. Reifenberger G, Liu L, Ichimura K, Schmidt EE, Collins VP. Amplification and overexpression of the MDM2 gene in a subset of human malignant gliomas without p53 mutations. Cancer Res. 1993;53:2736-9.

7. Verhaak RGW, Hoadley KA, Purdom E, Wang V, Qi Y, Wilkerson MD, Miller CR, Ding L, Golub T, Mesirov JP, et al. An integrated genomic analysis identifies clinically relevant subtypes of glioblastoma characterized by abnormalities in PDGFRA, IDH1, EGFR and NF1. Cancer Cell. 2010;17:98.

8. Wang Q, Hu B, Hu X, Kim H, Squatrito M, Scarpace L, AC dC, Lyu S, Li P, Li Y, et al. Tumor evolution of gliomaintrinsic gene expression subtypes associates with immunological changes in the microenvironment. Cancer Cell. 2017:32:42-56.e46.

9. Korber V, Yang J, Barah P, Wu Y, Stichel D, Gu Z, Fletcher MNC, Jones D, Hentschel B, Lamszus K, et al. Evolutionary trajectories of IDH (WT) glioblastomas reveal a common path of early tumorigenesis instigated years ahead of initial diagnosis. Cancer Cell. 2019;35:692-704.e612.

10. Kim H, Zheng S, Amini SS, Virk SM, Mikkelsen T, Brat DJ, Grimsby J, Sougnez C, Muller F, Hu J, et al. Whole-genome and multisector exome sequencing of primary and post-treatment glioblastoma reveals patterns of tumor evolution. Genome Res. 2015;25:316-27.

11. Cuykendall TN, Rubin MA, Khurana E. Non-coding genetic variation in cancer. Curr Opin Syst Biol. 2017;1:9-15.

12. Lindblad-Toh K, Garber M, Zuk O, Lin MF, Parker BJ, Washietl S, Kheradpour P, Ernst J, Jordan G, Mauceli E, et al. A highresolution map of human evolutionary constraint using 29 mammals. Nature. 2011;478:476-82

13. Davydov EV, Goode DL, Sirota M, Cooper GM, Sidow A, Batzoglou S. Identifying a high fraction of the human genome to be under selective constraint using GERP++. PLoS Comput Biol. 2010;6:e1001025.

14. RNAcentral: a hub of information for non-coding RNA sequences. Nucleic Acids Res 2019, 47:D1250-d1251.

15. Fishilevich S, Nudel R, Rappaport N, Hadar R, Plaschkes I, Iny Stein T, Rosen N, Kohn A, Twik M, Safran M, et al. GeneHancer: genome-wide integration of enhancers and target genes in GeneCards. Database (Oxford). 2017;2017:1-17.

16. Casper J, Zweig AS, Villarreal C, Tyner C, Speir ML, Rosenbloom KR, Raney BJ, Lee CM, Lee BT, Karolchik D, et al. The UCSC Genome Browser database: 2018 update. Nucleic Acids Res. 2018;46:D762-d769.

17. International Human Genome Sequencing C, Lander ES, Linton LM, Birren B, Nusbaum C, Zody MC, Baldwin J, Devon K, Dewar K, Doyle M, et al. Initial sequencing and analysis of the human genome. Nature. 2001;409:860.

18. Raine KM, Van Loo P, Wedge DC, Jones D, Menzies A, Butler AP, Teague JW, Tarpey P, Nik-Zainal S, Campbell PJ. ascatNgs: identifying somatically acquired copy-number alterations from whole-genome sequencing data. Curr Protoc Bioinformatics. 2016;56:15.19.11-7.

19. Beroukhim R, Mermel CH, Porter D, Wei G, Raychaudhuri S, Donovan J, Barretina J, Boehm JS, Dobson J, Urashima M, et al. The landscape of somatic copy-number alteration across human cancers. Nature. 2010;463:899-905.

20. Freire P, Vilela M, Deus H, Kim Y-W, Koul D, Colman H, Aldape KD, Bogler O, Yung WKA, Coombes K, et al. Exploratory analysis of the copy number alterations in glioblastoma multiforme. PLoS One. 2008;3:e4076. 
21. Cibulskis K, Lawrence MS, Carter SL, Sivachenko A, Jaffe D, Sougnez C, Gabriel S, Meyerson M, Lander ES, Getz G. Sensitive detection of somatic point mutations in impure and heterogeneous cancer samples. Nat Biotechnol. 2013;31:213-9.

22. Saunders CT, Wong WS, Swamy S, Becq J, Murray LJ, Cheetham RK. Strelka: accurate somatic small-variant calling from sequenced tumor-normal sample pairs. Bioinformatics. 2012;28:1811-7.

23. Ramos AH, Lichtenstein L, Gupta M, Lawrence MS, Pugh TJ, Saksena G, Meyerson M, Getz G. Oncotator: cancer variant annotation tool. Hum Mutat. 2015;36:E2423-9.

24. Lawrence MS, Stojanov P, Polak P, Kryukov GV, Cibulskis K, Sivachenko A, Carter SL, Stewart C, Mermel CH, Roberts SA, et al. Mutational heterogeneity in cancer and the search for new cancer-associated genes. Nature. 2013:499:214-8

25. Grossman RL, Heath AP, Ferretti V, Varmus HE, Lowy DR, Kibbe WA, Staudt LM. Toward a shared vision for cancer genomic data. N Engl J Med. 2016;375:1109-12.

26. Forbes SA, Bindal N, Bamford S, Cole C, Kok CY, Beare D, Jia M, Shepherd R, Leung K, Menzies A, et al. COSMIC: mining complete cancer genomes in the catalogue of somatic mutations in cancer. Nucleic Acids Res. 2011;39:D945-50. https://academic.oup.com/nar/article/39/suppl_1/D945/2508687.

27. Cerami E, Gao J, Dogrusoz U, Gross BE, Sumer SO, Aksoy BA, Jacobsen A, Byrne C, Heuer ML, Larsson E, et al. The cBio cancer genomics portal: an open platform for exploring multidimensional cancer genomics data. Cancer Discov. 2012;2:401-4.

28. Chang MT, Bhattarai TS, Schram AM, Bielski CM, Donoghue MTA, Jonsson P, Chakravarty D, Phillips S, Kandoth C, Penson A, et al. Accelerating discovery of functional mutant alleles in cancer. Cancer Discov. 2018:8:174-83.

29. Fan Y, Lee S, Wu G, Easton J, Yergeau D, Dummer R, Vogel P, Kirkwood JM, Barnhill RL, Pappo A, Bahrami A. Telomerase expression by aberrant methylation of the TERT promoter in melanoma arising in giant congenital nevi. J Invest Dermatol. 2016;136:339-42.

30. Borah S, Xi L, Zaug AJ, Powell NM, Dancik GM, Cohen SB, Costello JC, Theodorescu D, Cech TR. Cancer. TERT promoter mutations and telomerase reactivation in urothelial cancer. Science (New York). 2015;347:1006-10.

31. Heidenreich B, Rachakonda PS, Hosen I, Volz F, Hemminki K, Weyerbrock A, Kumar R. TERT promoter mutations and telomere length in adult malignant gliomas and recurrences. Oncotarget. 2015;6:10617-33.

32. Forbes SA, Beare D, Gunasekaran P, Leung K, Bindal N, Boutselakis H, Ding M, Bamford S, Cole C, Ward S, et al. COSMIC: exploring the world's knowledge of somatic mutations in human cancer. Nucleic Acids Res. 2015;43:D805-11.

33. Khurana E, Fu Y, Chakravarty D, Demichelis F, Rubin MA, Gerstein M. Role of non-coding sequence variants in cancer. Nat Rev Genet. 2016;17:93-108.

34. Battle A, Mostafavi S, Zhu X, Potash JB, Weissman MM, McCormick C, Haudenschild CD, Beckman KB, Shi J, Mei R, et al. Characterizing the genetic basis of transcriptome diversity through RNA-sequencing of 922 individuals. Genome Res. 2014;24:14-24

35. Wittkopp PJ, Kalay G. Cis-regulatory elements: molecular mechanisms and evolutionary processes underlying divergence. Nat Rev Genet. 2011;13:59-69.

36. Albert FW, Kruglyak L. The role of regulatory variation in complex traits and disease. Nat Rev Genet. 2015;16:197-212.

37. Davis CA, Hitz BC, Sloan CA, Chan ET, Davidson JM, Gabdank I, Hilton JA, Jain K, Baymuradov UK, Narayanan AK, et al. The Encyclopedia of DNA elements (ENCODE): data portal update. Nucleic Acids Res. 2018;46:D794-d801.

38. Man J, Shoemake J, Zhou W, Fang X, Wu Q, Rizzo A, Prayson R, Bao S, Rich JN, Yu JS. Sema3C promotes the survival and tumorigenicity of glioma stem cells through Rac1 activation. Cell Rep. 2014;9:1812-26.

39. Thomas-Chollier M, Hufton A, Heinig M, O'Keeffe S, Masri NE, Roider HG, Manke T, Vingron M. Transcription factor binding predictions using TRAP for the analysis of ChIP-seq data and regulatory SNPs. Nat Protoc. 2011;6:1860-9.

40. Neubauer HA, Tea MN, Zebol JR, Gliddon BL, Stefanidis C, Moretti PAB, Pitman MR, Costabile M, Kular J, Stringer BW, et al. Cytoplasmic dynein regulates the subcellular localization of sphingosine kinase 2 to elicit tumor-suppressive functions in glioblastoma. Oncogene. 2019;38:1151-65.

41. Yap YL, Lam DC, Luc G, Zhang XW, Hernandez D, Gras R, Wang E, Chiu SW, Chung LP, Lam WK, et al. Conserved transcription factor binding sites of cancer markers derived from primary lung adenocarcinoma microarrays. Nucleic Acids Res. 2005;33:409-21.

42. Maunakea AK, Nagarajan RP, Bilenky M, Ballinger TJ, D'Souza C, Fouse SD, Johnson BE, Hong C, Nielsen C, Zhao Y, et al. Conserved role of intragenic DNA methylation in regulating alternative promoters. Nature. 2010;466:253-7.

43. Wang Z, Yang B, Zhang M, Guo W, Wu Z, Wang Y, Jia L, Li S, Xie W, Yang D. IncRNA epigenetic landscape analysis identifies EPIC1 as an oncogenic IncRNA that interacts with MYC and promotes cell-cycle progression in cancer. Cancer Cell. 2018;33:706-720.e709.

44. Bralten LBC, Gravendeel AM, Kloosterhof NK, Sacchetti A, Vrijenhoek T, Veltman JA, van den Bent MJ, Kros JM, Hoogenraad CC, Sillevis Smitt PAE, French PJ. The CASPR2 cell adhesion molecule functions as a tumor suppressor gene in glioma. Oncogene. 2010;29:6138.

45. Hu B, Wang Q, Wang YA, Hua S, Sauve CG, Ong D, Lan ZD, Chang Q, Ho YW, Monasterio MM, et al. Epigenetic activation of WNT5A drives glioblastoma stem cell differentiation and invasive growth. Cell. 2016;167:1281-1295.e1218.

46. Li X, Zhang H, WU X. Long noncoding RNA DLX6-AS1 accelerates the glioma carcinogenesis by competing endogenous sponging miR-197-5p to relieve E2F1. Gene. 2019;686:1-7.

47. Bozdag S, Li A, Riddick G, Kotliarov Y, Baysan M, Iwamoto FM, Cam MC, Kotliarova S, Fine HA. Age-specific signatures of glioblastoma at the genomic, genetic, and epigenetic levels. PLoS One. 2013;8:e62982.

48. Zhang C, Yang M, Li Y, Tang S, Sun X. FOXA1 is upregulated in glioma and promotes proliferation as well as cell cycle through regulation of cyclin D1 expression. Cancer Manag Res. 2018;10:3283-93.

49. Cheung AK, Lung HL, Ko JM, Cheng Y, Stanbridge EJ, Zabarovsky ER, Nicholls JM, Chua D, Tsao SW, Guan XY, Lung ML. Chromosome 14 transfer and functional studies identify a candidate tumor suppressor gene, mirror image polydactyly 1, in nasopharyngeal carcinoma. Proc Natl Acad Sci U S A. 2009;106:14478-83.

50. Xiao Q, Sun Y, Dobi A, Srivastava S, Wang W, Srivastava S, Ji Y, Hou J, Zhao G-P, Li Y, Li H. Systematic analysis reveals molecular characteristics of ERG-negative prostate cancer. Sci Rep. 2018;8:12868.

51. Lee J, Lee AJ, Lee JK, Park J, Kwon Y, Park S, Chun H, Ju YS, Hong D. Mutalisk: a web-based somatic MUTation AnaLylS toolKit for genomic, transcriptional and epigenomic signatures. Nucleic Acids Res. 2018;46:W102-w108.

52. Alexandrov LB, Nik-Zainal S, Wedge DC, Aparicio SA, Behjati S, Biankin AV, Bignell GR, Bolli N, Borg A, Borresen-Dale AL, et al. Signatures of mutational processes in human cancer. Nature. 2013;500:415-21. 
53. Rands CM, Meader S, Ponting CP, Lunter G. $8.2 \%$ of the human genome is constrained: variation in rates of turnover across functional element classes in the human lineage. PLoS Genet. 2014;10:e1004525.

54. Koonin EV, Wolf YI. Constraints and plasticity in genome and molecular-phenome evolution. Nat Rev Genet. 2010;11:487.

55. Hui DHF, Tam KJ, Jiao IZF, Ong CJ. Semaphorin $3 \mathrm{C}$ as a therapeutic target in prostate and other cancers. Int J Mol Sci. 2019;20. https://www.mdpi.com/about/announcements/784

56. Hao J, Yu JS. Semaphorin 3C and its receptors in cancer and cancer stem-like cells. Biomedicines. 2018;6. https:/www. mdpi.com/about/announcements/784.

57. Smith AJ, Humphries SE. Characterization of DNA-binding proteins using multiplexed competitor EMSA. J Mol Biol. 2009; 385:714-7.

58. Lentjes MH, Niessen HE, Akiyama Y, de Bruine AP, Melotte V, van Engeland M. The emerging role of GATA transcription factors in development and disease. Expert Rev Mol Med. 2016;18:e3.

59. Zheng R, Blobel GA. GATA transcription factors and cancer. Genes Cancer. 2010;1:1178-88.

60. Wang Z, Yuan H, Sun C, Xu L, Chen Y, Zhu Q, Zhao H, Huang Q, Dong J, Lan Q. GATA2 promotes glioma progression through EGFR/ERK/Elk-1 pathway. Med Oncol. 2015;32:87.

61. Glimelius B, Melin B, Enblad G, Alafuzoff I, Beskow A, Ahlstrom H, Bill-Axelson A, Birgisson H, Bjor O, Edqvist PH, et al. UCAN: a prospective longitudinal collection of biomaterials and clinical information from adult cancer patients in Sweden. Acta Oncol. 2018;57:187-94.

62. Xie Y, Bergström T, Jiang Y, Johansson P, Marinescu VD, Lindberg N, Segerman A, Wicher G, Niklasson M, Baskaran S, et al. The human glioblastoma cell culture resource: validated cell models representing all molecular subtypes. EBioMedicine. 2015;2:1351-63.

63. Li H, Durbin R. Fast and accurate short read alignment with Burrows-Wheeler transform. Bioinformatics. 2009;25:1754-60.

64. McKenna A, Hanna M, Banks E, Sivachenko A, Cibulskis K, Kernytsky A, Garimella K, Altshuler D, Gabriel S, Daly M, DePristo MA. The Genome Analysis Toolkit: a MapReduce framework for analyzing next-generation DNA sequencing data. Genome Res. 2010;20:1297-303.

65. Sherry ST, Ward MH, Kholodov M, Baker J, Phan L, Smigielski EM, Sirotkin K. dbSNP: the NCBI database of genetic variation. Nucleic Acids Res. 2001;29:308-11.

66. Ameur A, Dahlberg J, Olason P, Vezzi F, Karlsson R, Martin M, Viklund J, Kähäri AK, Lundin P, Che H, et al. SweGen: a whole-genome data resource of genetic variability in a cross-section of the Swedish population. Eur J Hum Genet. 2017; 25:1253.

67. Sakthikumar S, Roy A, Haseeb L, Pettersson ME, Sundström E, Marinescu VD, Lindblad-Toh K, Forsberg-Nilsson, K. Whole genome sequencing of glioblastoma reveals enrichment of non-coding constraint mutations in known and novel genes. Dataset. European Genome-Phenome Archive. https://www.ega-archive.org. Accessed 3 June 2020.

\section{Publisher's Note}

Springer Nature remains neutral with regard to jurisdictional claims in published maps and institutional affiliations.

Ready to submit your research? Choose BMC and benefit from:
- fast, convenient online submission
- thorough peer review by experienced researchers in your field
- rapid publication on acceptance
- support for research data, including large and complex data types
- gold Open Access which fosters wider collaboration and increased citations
- maximum visibility for your research: over 100M website views per year
At BMC, research is always in progress.
Learn more biomedcentral.com/submissions

